\title{
Alzheimer's Disease Genes Are Associated with Measures of Cognitive Ageing in the Lothian Birth Cohorts of 1921 and 1936
}

\author{
Gillian Hamilton, ${ }^{1}$ Sarah E. Harris, ${ }^{1,2}$ Gail Davies, ${ }^{3}$ David C. Liewald, ${ }^{3}$ Albert Tenesa, ${ }^{4}$ \\ John M. Starr, ${ }^{2,5}$ David Porteous, ${ }^{1,2}$ and Ian J. Deary ${ }^{2,3}$ \\ ${ }^{1}$ Medical Genetics, Molecular Medicine Centre, University of Edinburgh, Crewe Road South, Edinburgh EH4 2XU, UK \\ ${ }^{2}$ Centre for Cognitive Ageing and Cognitive Epidemiology, University of Edinburgh, 7 George Square, Edinburgh EH8 9JZ, UK \\ ${ }^{3}$ Department of Psychology, University of Edinburgh, 7 George Square, Edinburgh EH8 9JZ, UK \\ ${ }^{4}$ MRC Human Genetics Unit, The Institute of Genetics and Molecular Medicine, Western General Hospital, \\ Edinburgh EH4 2XU, UK \\ ${ }^{5}$ Geriatric Medicine Unit, Royal Victoria Hospital, University of Edinburgh, Craigleith Road, Edinburgh EH4 2DN, UK
}

Correspondence should be addressed to Ian J. Deary, iand@staffmail.ed.ac.uk

Received 24 November 2010; Revised 23 February 2011; Accepted 27 March 2011

Academic Editor: Lars Bertram

Copyright ( 2011 Gillian Hamilton et al. This is an open access article distributed under the Creative Commons Attribution License, which permits unrestricted use, distribution, and reproduction in any medium, provided the original work is properly cited.

\begin{abstract}
Alzheimer's disease patients have deficits in specific cognitive domains, and susceptibility genes for this disease may influence human cognition in nondemented individuals. To evaluate the role of Alzheimer's disease-linked genetic variation on cognition and normal cognitive ageing, we investigated two Scottish cohorts for which assessments in major cognitive domains are available: the Lothian Birth Cohort of 1921 and the Lothian Birth Cohort of 1936, consisting of 505 and 998 individuals, respectively. 158 SNPs from eleven genes were evaluated. Single SNP analyses did not reveal any statistical association after correction for multiple testing. One haplotype from TRAPPC6A was associated with nonverbal reasoning in both cohorts and combined data sets. This haplotype explains a small proportion of the phenotypic variability (1.8\%). These findings warrant further investigation as biological modifiers of cognitive ageing.
\end{abstract}

\section{Introduction}

Alzheimer's disease (AD) is the most common neurodegenerative disease, and it is predicted to affect over a million people in the UK by 2025 (Dementia UK 2007 report). AD is characterised initially by impaired episodic memory [1] and, as the disease progresses, other cognitive deficits appear, particularly in attention and executive functions, semantic memory, language, and spatial orientation $[2,3]$.

$\mathrm{AD}$ is a genetically heterogeneous disease. Mutations in three genes (the amyloid precursor protein, APP; presenilin 1, PS1; presenilin 2, PS2) are known to cause a rare earlyonset form of $\mathrm{AD}$ [4-6]. The most common form of $\mathrm{AD}$ occurs sporadically and with a late age at onset. Until recently, the only well-replicated risk factor for this form of $\mathrm{AD}$ was the $\varepsilon 4$ allele of the apolipoprotein $\mathrm{E}(A P O E)$ gene [7]. However, three recent genome-wide association studies (GWASs) have identified four new candidate genes for sporadic AD_BIN1, CLU, CR1, and PICALM-and one new genomic region near BLOC1S3/EXOC3L2/MARK4 [810]. Associations with CLU, CR1, and PICALM have been replicated [11-13].

Nonpathological age-related cognitive decline is a major and growing concern in developed societies [14]. General cognitive ability is an important predictor of life outcomes, including in old age. The determinants of normal cognitive ageing are not fully understood, but are likely to include both genetic and environmental influences [14]. Genetic influences on cognitive ability increase from about $30 \%$ in childhood to as much as $80 \%$ in later adulthood, and these decrease slightly in very old-age when, probably, stochastic effects become relatively more important [15]. As is still 
TABLe 1: Details of the Lothian Birth Cohorts of 1921 and 1936.

\begin{tabular}{lcc}
\hline & LBC1921 & LBC1936 \\
\hline Total & 505 & 998 \\
Females $(\%)$ & $296(58.7)$ & $494(49.5)$ \\
Males $(\%)$ & $209(41.3)$ & $504(50.5)$ \\
Mean age in years ${ }^{1}( \pm$ s.d) & $10.9 \pm 0.28$ & $10.9 \pm 0.28$ \\
Mean age in years ${ }^{2}( \pm$ s.d) & $79.11 \pm 0.57$ & $69.58 \pm 0.83$ \\
$\geq 1$ APOE $\varepsilon 4$ allele & 135 & 287 \\
no APOE $\varepsilon 4$ allele & 370 & 672 \\
\hline
\end{tabular}

${ }^{1}$ Mean age at original test date, ${ }^{2}$ Mean age when revisited.

true for many complex phenotypes, there are few replicated genotype-phenotype associations with cognitive ageing [15, 16]. There is suggestive evidence for genes such as BDNF and $C O M T$ but, to date, $A P O E$ is the only gene that has been consistently shown to have a significant, but small, influence on age-related cognitive decline [17]. We hypothesise that other genes involved in $\mathrm{AD}$ may play a role in normal cognitive ageing. Indeed, a recent study has described the association of variants in the CLU and PICALM genes with cognitive function [18].

Here, we examine genetic variants from the APP, PS1, PS2, BIN1, CLU, CR1, PICALM genes, and the region surrounding the BLOC1S3/EXOC3L2/MARK4 genes on chromosome 19 in two large, phenotypically well-defined cohorts, the Lothian Birth Cohorts of 1921 and 1936 [19, 20]. The individuals in these cohorts took a general mental ability test in childhood and then took a range of mental tests in old age. They are, therefore, unusually useful in understanding the genetic contributions to cognitive change across most of the human life course. The APOE gene has previously been investigated in these cohorts and shown to explain a small percentage $(0.005-0.01)$ of the variance associated with the general cognitive factor, two nonverbal tests, and choice reaction time variability [21-24].

\section{Materials and Methods}

2.1. Sample. The samples examined were the Lothian Birth Cohort of 1921 (LBC1921) and the Lothian Birth Cohort of 1936 (LBC1936). They were born in 1921 and 1936, respectively and, at a mean age of 11 years, they were tested on general cognitive ability by means of the Scottish Mental Survey of 1932 (SMS1932) or the Scottish Mental Survey of 1947 (SMS1947) (each cohort has a mean age $=10.9 \pm$ 0.28 years). Since 1999 for LBC1921 and 2004 for LBC1936, a number of the original Surveys' participants who were living in the Edinburgh area of Scotland have been revisited. Participants from LBC1921 were tested for a variety of cognitive phenotypes at approximately 79 years of age (mean age $=79.11 \pm 0.57$ years), whereas participants from the LBC1936 were tested at approximately 70 years of age (mean age $=69.58 \pm 0.83$ years $)($ Table 1$)[19,20]$.

Individuals were excluded from this study if there was a personal history of dementia, if they had an MMSE score of less than 24, or if they did not have GWAS data. Four individuals were removed from the LBC1921 due to a family history of dementia, and eight were removed due to MMSE $<24$. Seven individuals were removed from the LBC1936 with MMSE $<24$. The total number of participants included from the LBC1921 was 505 (41.3\% male: 58.7\% female), and the total number of participants from the LBC1936 was 998 (50.5\% male: $49.5 \%$ female) (Table 1 ).

The LBC1936 was used as the discovery cohort. Significant results meeting the chosen statistical criteria were carried forward and investigated using the LBC1921.

2.2. Cognitive Tests. Individuals from the LBC1936 were tested on the Moray House Test (MHT) no. 12 at age 11 $(10.9 \pm 0.28$ years $)$ and subsequently at age $70(69.58 \pm 0.83$ years) [19]. At age 70, they were also tested for a variety of cognitive phenotypes, with the ones of interest to this study being verbal fluency (a test of executive function using the letters $\mathrm{C}, \mathrm{F}$, and $\mathrm{L}$ ) [25], matrix reasoning (a subtest from the Wechsler Adult Intelligence Scale-IIIUK ${ }^{\mathrm{UK}}$ used to assess nonverbal reasoning) [26], and logical memory (a test of immediate and delayed verbal declarative memory from the Wechsler Memory Scale-III ${ }^{\mathrm{UK}}$ ) [27].

Individuals from the LBC1921 were tested on the MHT no. 12 at age $11(10.9 \pm 0.28$ years $)$ and subsequently at age 79 (79.11 \pm 0.57 years) [20]. This cohort's participants were tested for three cognitive phenotypes; verbal fluency (exactly as applied in the LBC1936), Raven's Standard Progressive Matrices (a test of non-verbal reasoning) [28], and logical memory (a test of immediate and delayed verbal declarative memory from the Wechsler Memory Scale-Revised [29].

From this point forward, age 11 for both cohorts indicates $10.9 \pm 0.28$; age 70 for the LBC1936 cohort indicates $69.58 \pm 0.83$ years; age 79 for the LBC1921 cohort indicates $79.11 \pm 0.57$ years.

2.3. Genotyping. Genomic DNA from the LBC1936 cohort was isolated from whole blood by standard procedures at the Wellcome Trust Clinical Research Facility (WTCRF), Genetics Core, Western General Hospital, Edinburgh. Genomic DNA from the LBC1921 cohort was isolated from whole blood by standard procedures at Medical Research Council (MRC) Technology, Western General Hospital, Edinburgh. All samples were genotyped at the WTCRF Genetics Core with the Illumina Human 610-Quadv1 chip as part of a larger study [30]. SNPs were included in the analyses if they met the following conditions: call rate $\geq 0.98$, minor allele frequency $\geq 0.01$, and Hardy-Weinberg Equilibrium test with $P \geq .001$ [30]. For this study, specific SNPs were selected from the GWAS data set. Genomic regions approximately $5 \mathrm{~kb}$ upstream to $5 \mathrm{~kb}$ downstream of each candidate gene were identified using positional information from the Santa Cruz Genome Browser, March 2006 Assembly (NCBI36) (http://genome.ucsc.edu/) [31]. All SNPs with available genotype data from each region were used in this study. A further five SNPs that showed association with sporadic $\mathrm{AD}$ were included: four that were outside the above genomic regions and one that was within the genomic region but that had not been genotyped. This SNP (rs6656401) 
was imputed using the HapMap phase II CEU data (NCBI build 36 (UCSC hg18)) as the reference sample and MACH software. The imputation quality score for this SNP was high $\left(r^{2}=0.92\right)$. A total of 158 SNPs were selected; 66 from APP, 9 from PS1, 6 from PS2, 17 from BIN1, 6 from CLU, 9 from CR1, 29 from PICALM, and 16 from the $B L O C 1 S 3 / E X O C 3 L 2 / M A R K 4$ region, which included three SNPs from the $5^{\prime}$ end of TRAPPC6A gene (Table S1). APOE haplotype data were available for all samples.

\subsection{Statistical Analysis}

2.4.1. Significance Threshold. To determine the correct level of significance for regression and haplotype analyses of the LBC1936 cohort, a spectral decomposition program, SNPSpD, was used [9]. SNPSpD calculates an approximate estimate of the effective number of independent SNPs using a previously described method [32]. A Bonferroni calculation using this number of SNPs was used to determine the appropriate level of significance for regression and haplotype analysis. A significance level for pairwise interaction analyses of the LBC1936 cohort was determined using $\alpha=0.05 / x$, where $x=n(n-1) / 2(n=$ effective number of independent SNPs) [33].

2.4.2. Cognitive Phenotypes. Standardized residual scores were calculated for each cognitive phenotype to incorporate age at time of testing and gender, using linear regression in SPSS, v14.0.

2.4.3. Association Analysis. Unless otherwise noted, all statistical analyses were carried out using PLINK v1.07 (http:// pngu.mgh.harvard.edu/purcell/plink) [34]. Three approaches to association analysis were used.

The first approach examined all SNPs in relation to the selected cognitive phenotypes and applied a stringent Bonferonni threshold to the $P$ values. Linear regression analysis was performed under an additive model in PLINK. Additional analyses included two covariates; (i) the presence or absence of an $A P O E \& 4$ allele and (ii) general cognitive ability at age 11 (MHT score adjusted for age) to adjust for prior cognitive ability. Using general cognitive ability at age 11 as a covariate enables the role of each SNP in cognitive ageing to be explored. Two stratified data sets, with or without the APOE $\& 4$ allele, were analysed similarly. Adaptive permutation analysis was carried out on all linear regression analyses.

The second approach was haplotype analysis. Each gene was examined for association with cognitive phenotypes using a sliding window of three SNPs, shifting one SNP at a time. Two stratified data sets, with or without the APOE $\varepsilon 4$ allele, were analysed similarly. In the haplotype analysis, the presence or absence of an $A P O E$ \&4 allele and general cognitive ability at age 11 (MHT score adjusted for age) were not used as covariates. SNP regions meeting the significance threshold were analysed using $\max (\mathrm{T})$, a label swappingbased permutation method.
The third and final approach used pairwise interaction analysis to determine any effect of gene-gene interaction on the association with cognitive phenotypes. The full data set and two stratified data sets, with or without the $A P O E \& 4$ allele, were analysed similarly. In the pairwise interaction analysis, the presence or absence of an APOE $\varepsilon 4$ allele and general cognitive ability at age 11 (MHT score adjusted for age) were not used as covariates. The results file was controlled so that only associations having $P \leq .0001$ were reported. Additionally, only where SNPs were located in different genes are the pairwise interactions described here. Significant interactions were analysed using a one-way ANOVA in SPSS v14.0. To examine each interaction, both the cognitive mean of each genotype (aabb, aaBB, aaBb, AAbb, $\mathrm{Aabb}, \mathrm{AABB}, \mathrm{AABb}, \mathrm{AaBB}, \mathrm{AaBb})$ and the cognitive mean of the groups representing the presence or absence of each minor allele (aabb, aaB-, A-bb, A-B-) were compared (where $a$ and $b$ represent the minor allele of each $\mathrm{SNP}$ ).

2.4.4. Linkage Disequilibrium Analysis. Linkage disequilibrium (LD) values were generated and visualised using Haploview [35].

\section{Results}

3.1. Significance Threshold. 158 SNPs in total were selected for analysis in this study (Table S1 in Supplementary Material available online at doi:10.4061/2011/505984). The LBC1936 cohort was used as a discovery sample and the LBC1921 cohort as a replication cohort. Different significance thresholds were applied to each cohort. To determine an appropriate threshold for analyses of the discovery cohort, two methods were used. Spectral decomposition analysis calculated that the approximate estimate of the effective number of independent SNPs was 89.24. Therefore, in our regression and haplotype analyses, only where $P \leq$ $.00056(\alpha=0.05 / 89.24)$, were results considered significant associations. For pairwise interaction analysis, only where $P \leq .000013(\alpha=0.05 / \mathrm{x}, \mathrm{x}=[89.24(89.24-1)] / 2)$ were results considered significant associations. $\max (\mathrm{T})$ permutation analysis was carried out on significant haplotype results, and a significance threshold of $P \leq .05$ was applied to the results. Results with $P \leq .05$ were considered significant in our replication cohort.

3.2. Association of AD SNPs with Cognitive Phenotypes. No individual SNP in the LBC1936 was associated with any cognitive phenotype in the overall or APOE stratified sample at $P \leq .00056$ (Table S2, Table S3).

3.3. Association of AD Gene Blocks with Cognitive Phenotypes. Tables S4, S5, and S6 detail the effect of each 3-SNP window on each cognitive phenotype in the complete LBC1936 data set and in the LBC1936 data sets stratified for presence or absence of the $A P O E \varepsilon 4$ allele.

Two 3-SNP windows, comprising four adjacent SNPs from $B I N 1$, reached our corrected $P$ value level $(P \leq .00056)$ with general cognitive ability at age 11 (MHT adjusted) in 
the overall LBC1936 sample (Table 2). These results were not replicated in the LBC1921 and were nonsignificant following permutation analysis of both the LBC1936 and the combined data set.

Two separate 3-SNP windows from the APP locus reached significance with logical memory in the $A P O E$ $\varepsilon 4$ positive subgroup (Table 2 ). These SNP windows were not significant postpermutation analysis of the LBC1936. Further, this result was not replicated in the LBC1921 or following permutation analysis of the combined sample.

One 3-SNP window from the TRAPPC6A locus reached significance with matrix reasoning in the $A P O E \& 4$ negative subgroup (Table 2). Though not significant postpermutation analysis in the LBC1936, this finding was replicated in the LBC1921 and in post permutation analysis of the combined cohort.

3.4. Gene-Gene Interaction Analysis. Tables S7 11 detail the results obtained in the pairwise interaction analyses with each cognitive phenotype in the LBC1936. Data were extracted for interactions if $P \leq .0001$. Results were considered significant if $P \leq .000013$.

One SNP-SNP interaction from the chromosome 19 locus (MARK4, rs344807) and APP (rs12482753) was significantly associated with general cognitive ability at age 70 (MHT adjusted for age) in the APOE \&4 negative LBC1936 subset (Figure 1; Table 3 ). However, analysis of the cognitive means for each genotype group indicated that the association was due to the low score of a single individual who expressed the $\mathrm{a} \mathrm{ab}$ genotype. Analysis of the cognitive means of the four groups representing the presence or absence of the minor alleles showed no significant difference, and following the removal of the aaBb individual the genotype result was no longer significant (results not shown). This interaction was not replicated in the LBC1921.

A single SNP-SNP interaction from PS1 (rs214260) and $A P P$ (rs440666) was significantly associated with verbal fluency in the APOE $\varepsilon 4$ negative LBC1936 subset (Figure 1; Table 3). Analysis of the cognitive means for each genotype group indicated that the association was due to the lower verbal fluency scores of the group expressing the Aabb genotype; however, analysis of the cognitive means of the four groups representing the presence or absence of the minor alleles showed no significant difference (results not shown). This interaction was not replicated in the LBC1921.

One SNP-SNP interaction from BIN1 (rs10200967) and $A P P$ (rs2830036) was significantly associated with verbal declarative memory in the APOE \&4 positive LBC1936 subset (Figure 1; Table 3). Analysis of the cognitive means for each genotype and for the four groups representing the presence or absence of the minor alleles indicated that this association was due to the low logical memory scores of two individuals homozygous for each minor allele (Figure 2). Although not a direct replication of the result observed in the LBC1936 cohort, two BIN1-APP interactions approached significance in the LBC1921 cohort. The associations were observed with the BIN1 SNP (rs10200967) that was associated in the LBC1936 APOE $\varepsilon 4$ positive sample set, but with two different
APP SNPs: rs396969 and rs383700 (Table 3). The two APP SNPS were in complete LD (Figure 1). Both interactions were associated with higher logical memory scores, with the opposite of that observed in the LBC1936. Analysis of the cognitive means for each genotype indicated that both associations were due to the high logical memory score of one individual homozygous for each minor allele. Following the removal of this individual, this result was no longer significant (results not shown). No BIN1-APP SNP interactions were observed in the $A P O E \& 4$ positive samples in LBC1921, and there was no significant interaction when the samples were combined.

A single SNP-SNP interaction from PS2 (rs1150895) and PICALM (rs3851179) was significantly associated with verbal declarative memory in the APOE $\varepsilon 4$ negative LBC1936 subset (Figure 1; Table 3 ). Analysis of the cognitive means for each genotype group indicated that the association was due to the higher logical memory scores of the groups expressing either the AAbb or aaBB genotype compared to the AABB genotype. Further analysis of the cognitive means of the four groups representing the presence or absence of the minor alleles showed that $\mathrm{aaBB}$ and $\mathrm{aaBb}$ individuals had higher logical memory scores than other allele groups (results not shown). This interaction was not replicated in the LBC1921.

\section{Discussion}

In this study, we have screened polymorphisms from three causal and five putative risk genes for Alzheimer's disease in two cohorts with extensive and unique cognitive phenotypes available. Evidence was found to suggest a role for variation in a gene at the chromosome 19 locus, APP and BIN1 in cognitive ability.

Each gene will be discussed individually.

4.1. Chromosome 19 Locus. A genomic locus on chromosome 19 was recently implicated in a single LOAD-GWAS [10]. It identified a locus distal to and not in linkage disequilibrium with APOE. The SNPs chosen in this study span the $5^{\prime}$ end of the TRAPPC6A gene and cover BLOC1S3, EXOC3L2, $M A R K 4$, and the $3^{\prime}$ end of the CKM gene.

One 3-SNP window located at the $5^{\prime}$ end of the BLOC1S3/EXOC3L2/MARK4 region was significantly associated with non-verbal reasoning in individuals lacking an APOE \&4 gene in the LBC1936 data set. This SNP window consisted of the SNPs (rs7247764, rs28555639, rs12460041) located at the $5^{\prime}$ end of the TRAPPC6A gene. They span a genomic region of $1442 \mathrm{bp}$ and are in complete LD $\left(D^{\prime}=\right.$ $1)$. The genotype of this associated haplotype was TTT, and it was the most common haplotype $(f=0.70)$. This haplotype was associated with a small decrease in Wechsler matrix reasoning scores $(\beta=-0.21)$ and explained $1.8 \%$ of the variation in the LBC1936. This was replicated in the LBC1921 cohort $(\beta=-0.18)$, where it explained $1.3 \%$ of the variation in Raven's Standard Progressive Matrices scores. Permutation analysis of the combined data set confirmed this result. 


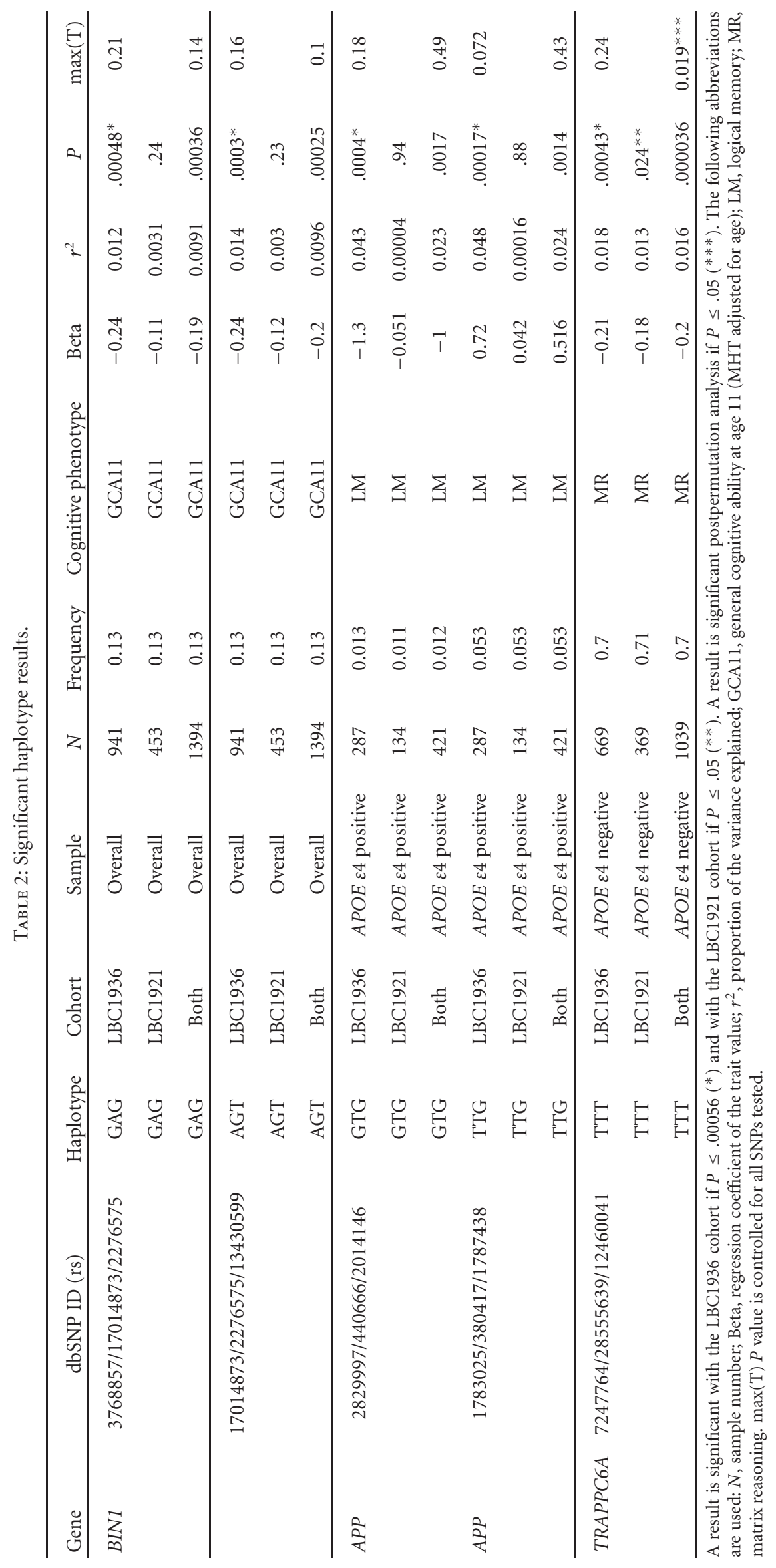




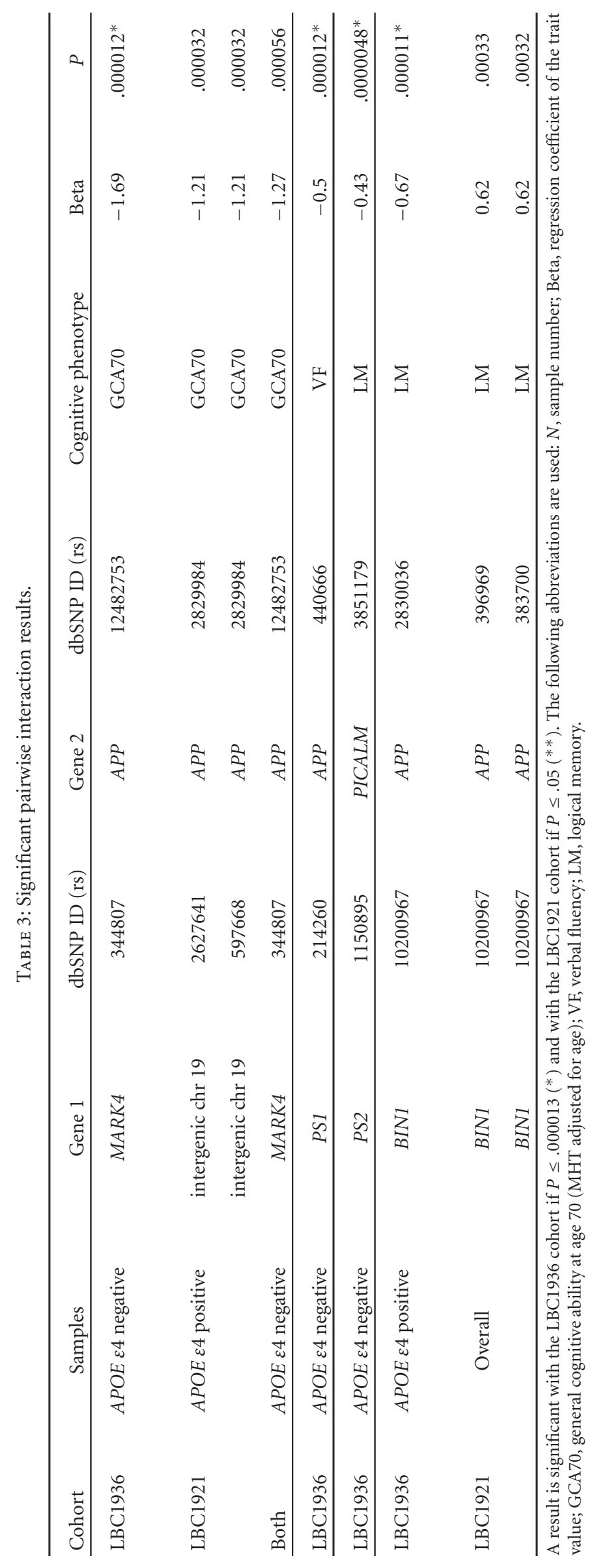




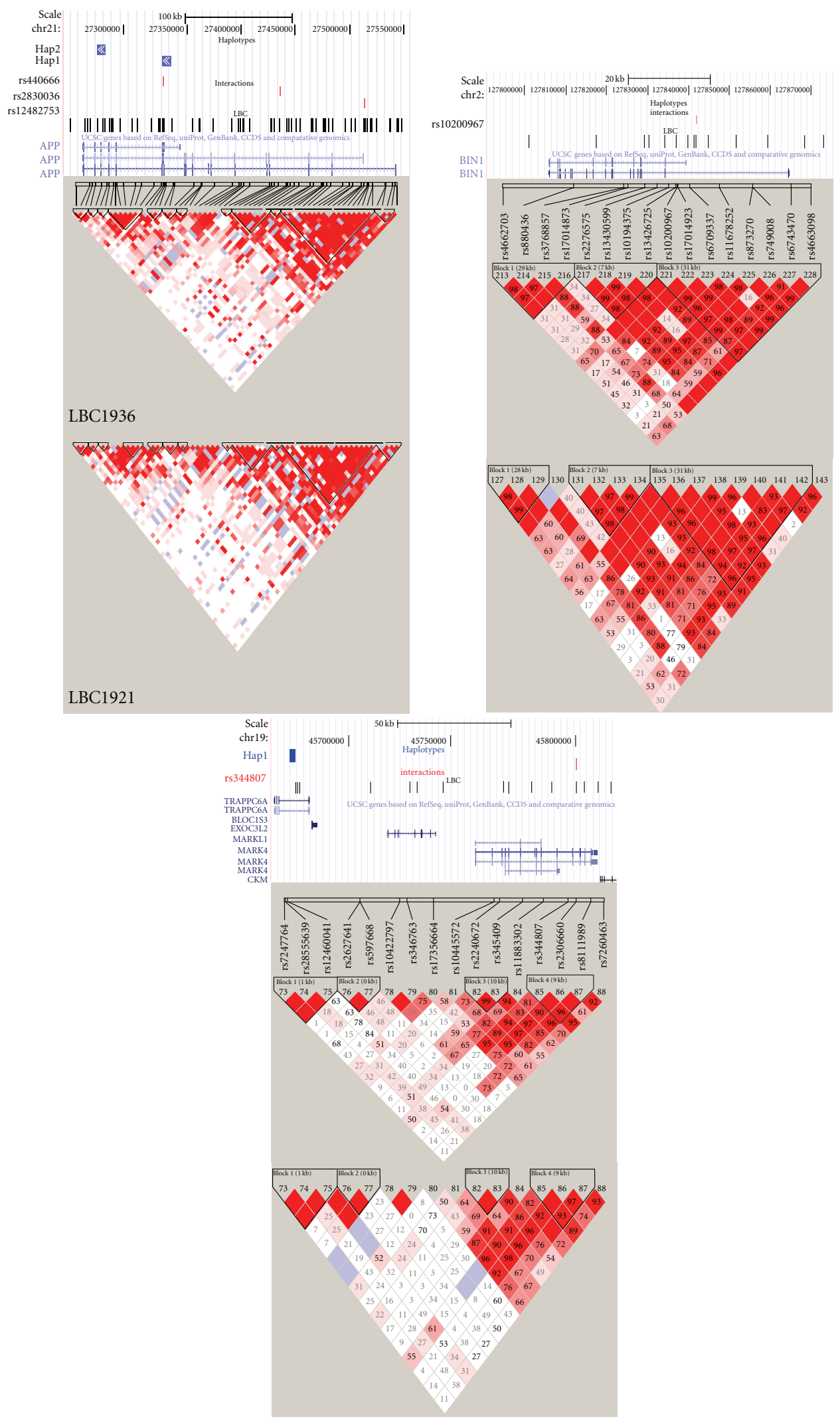

FIGURE 1: Genomic structure of positively associated genes. (a) Genomic structure of APP, BIN1, and chromosome 19. Highlighted are the location of each SNP genotyped and the location of positively associated haplotypes and gene-gene interactions. (b) LD structure of $A P P$, BIN1, and chromosome 19 in the Lothian Birth Cohorts of 1936 (top) and 1921 (bottom). LD values used were $D^{\prime}$. 


\begin{tabular}{|c|c|c|c|c|c|}
\hline & Sum of squares & $d f$ & Mean square & $F$ & Sig. \\
\hline Between groups & 21.611 & 8 & 2.701 & 2.96 & .003 \\
Within groups & 252.824 & 277 & .913 & & \\
Total & 274.434 & 285 & & & \\
\hline
\end{tabular}

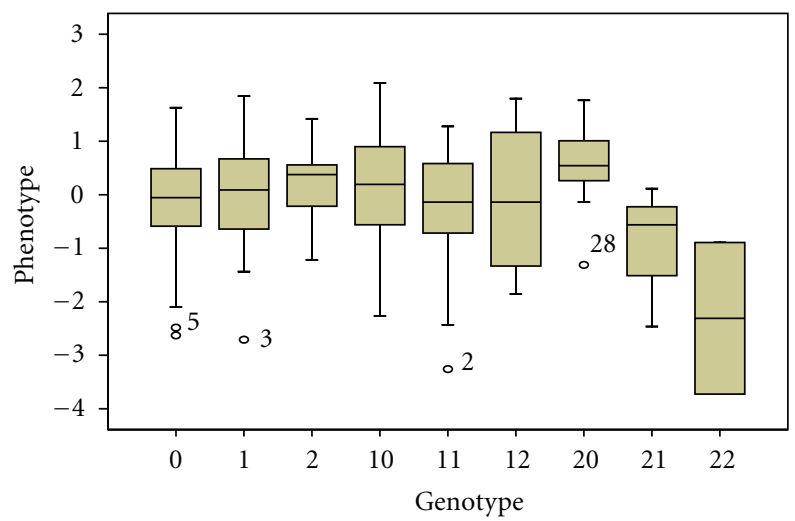

(a)

\begin{tabular}{|c|c|c|c|c|c|}
\hline & Sum of squares & $d f$ & Mean square & $F$ & Sig. \\
\hline Between groups & 11.58 & 3 & 3.86 & 4.141 & .007 \\
Within groups & 262.854 & 282 & .932 & & \\
Total & 274.434 & 285 & & & \\
\hline
\end{tabular}

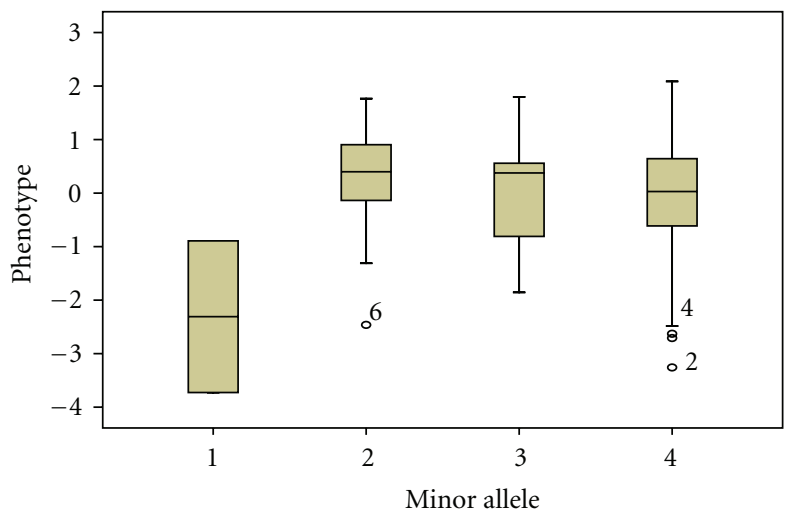

(b)

FIgURE 2: The interaction of an SNP pair from BIN1 and APP is likely to influence logical memory in the APOE $\varepsilon 4$ positive subset of LBC1936. Analysis of both the (a) genotype cognitive means and (b) the allele specific means shows that the initial positive result is due to two individuals carrying both minor alleles, aabb. Genotype legend; $11=\mathrm{AaBb}, 10=\mathrm{AaBB}, 01=\mathrm{AABb}, 00=\mathrm{AABB}, 12=\mathrm{Aabb}, 01=\mathrm{AAbb}$, $21=\mathrm{aaBb}, 20$ = aaBB, 22 = aabb. Allele legend; 1 = aabb, 2 = aaB-, 3 = A-bb, 4 = A-B-.

The SNP associated with LOAD in the recent GWAS study [10], rs597668, is located in an intergenic region between TRAPPC6A and EXOC3L2. This SNP was included in our study although we did not observe an association with any cognitive phenotype. The TRAPPC6A haplotype is located $31573 \mathrm{bp}$ from the GWAS SNP, and analysis of the $\mathrm{LD}$ in this region shows that SNPs from the haplotype were not in the same LD block as the GWAS SNP $\left(D^{\prime}=0.22\right)$, so it is unclear whether our results are detecting the same effect. Replication of the TRAPPC6A haplotype is required in a larger cohort.

4.2. APP. APP was the first disease gene identified in familial $\mathrm{AD}$ [4]. It is a transmembrane protein, and sequential cleavage by $\beta$ - and $\gamma$-secretase releases the $\beta$-amyloid peptide. Although the exact role of the APP protein is unknown, it is considered central to $\mathrm{AD}$ pathogenesis.

Two 3-SNP windows at the APP locus, each consisting of three SNPs, were associated with verbal declarative memory in individuals carrying at least one $A P O E \& 4$ allele in the LBC1936. These results correspond to two genomic regions located at the 3 ' end of the APP gene. The first region consisted of three SNPs, rs2829997, rs440666, and rs2014146, and spanned $8163 \mathrm{bp}$. These SNPs were in high LD $\left(D^{\prime}>0.7\right)$ and constituted a haplotype block. The associated haplotype, with genotype GTG, was rare, with a frequency of 0.013 . This haplotype, APP Hap1, was associated with a decrease in logical memory scores $(\beta=-1.312)$ and explained $4.3 \%$ of the variation. The second genomic region spanned $7326 \mathrm{bp}$ and consisted of three SNPs, rs1783025, rs380417, and rs1787438. These SNPs are located near known pathogenic
$\mathrm{AD}$ mutations, in sites encoding the $\alpha, \beta$, and $\gamma$-secretase sites. The latter two SNPs were in complete $\operatorname{LD}\left(D^{\prime}=1\right)$; however, rs1783025 was not $\left(D^{\prime} 0.48,0.64\right.$ with rs380417, rs1787438, respectively). The associated genotype, TTG, was rare, with a frequency of 0.053 . This genotype was associated with an increase in logical memory scores $(\beta=0.72)$ and explained $4.8 \%$ of the variation. These two genotypes explain a small, but important, amount of the variance, $4.3 \%$ and $4.8 \%$ respectively, especially considering that APOE $\varepsilon 4$ contributes $0.5-1 \%$ to variance in cognitive traits. However, these results were not replicated following permutation analysis. Further, this effect was not observed in the LBC1921 or in the combined data set.

These results may not have been replicated in the LBC1921 cohort for a couple of reasons: the replication cohort contains fewer individuals and the logical memory test used with the LBC1921 cohort differed slightly from that used with the LBC1936 cohort. Nonetheless, the haplotype frequencies are consistent between cohorts and, although not significant, LBC1921 individuals with APP Hap1 (GTG) have lower logical memory scores while individuals with the second associated genotype (TTG) have higher logical memory scores in the LBC1921.

Further evidence of a role for APP in logical memory was obtained in our gene-gene interaction analysis. SNPs at the APP locus were observed to statistically interact with polymorphisms at the BIN1 locus to influence verbal declarative memory.

4.3. BIN1. BIN1 was identified as a putative risk factor for LOAD in a recent GWAS study [10]. It encodes several 


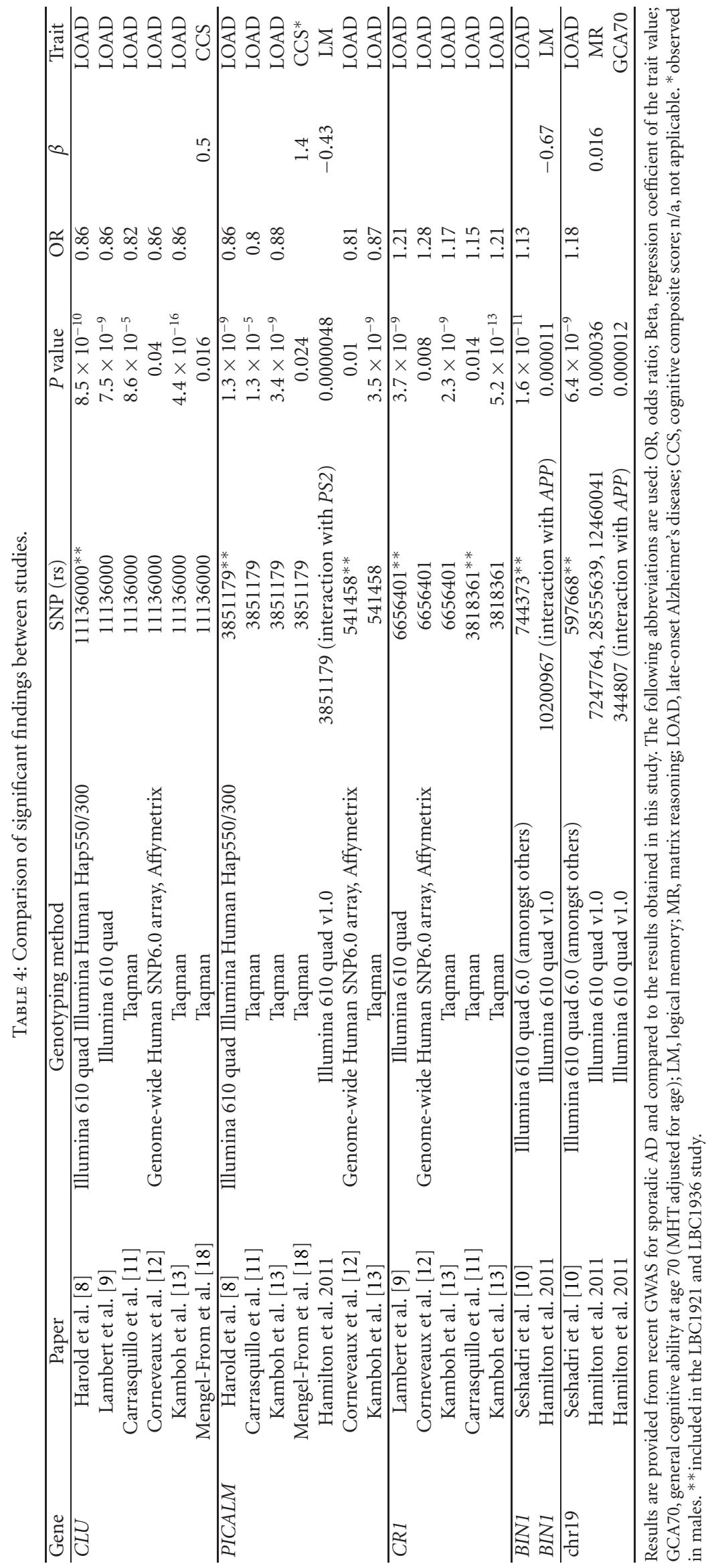


isoforms that are expressed in the central nervous system and may be involved in synaptic vesicle endocytosis.

An interaction between rs10200967 (BIN1) and rs2830036 $(A P P)$ was significantly associated with verbal declarative memory in the APOE \&4 positive LBC1936 subset. Further analysis showed that this was due to the low logical memory scores of two individuals expressing both minor alleles of rs10200967 (C, BIN1) and rs2830036 (T, APP) (Figure 2). This result was not replicated in the LBC1921 cohort or in the combined analysis.

However, these results are consistent with the association of APP Hap1, GTG, which is associated with a similar decrease in logical memory scores in the APOE $\varepsilon 4$ positive subset of LBC1936. The APP SNP involved in the APP-BIN1 interaction ( $\mathrm{rs} 2830036)$ is located $5^{\prime}$ to APP haplotype 1 but there are low levels of LD between them $\left(D^{\prime}=0.34\right)$. Indeed the two individuals contributing to the interaction association do not carry the APP Hapl genotype associated with a decrease in logical memory scores (APP Hap1 genotype, GTG; individual genotypes, both AA-CC-AG).

Although the BIN1-APP interaction was not replicated in the LBC1921, an association approaching significance was observed with variants from $A P P$ and BIN1 and verbal declarative memory in the overall LBC1921 cohort. This was due to the higher logical memory score of a single individual expressing both minor alleles of the two SNPs (BIN1, rs10200967; APP, rs396969 and rs383700), so may not hold up in a replication study. The two APP SNPs involved in this interaction were in $\operatorname{LD}\left(D^{\prime}=0.98\right)$ with the second APP region, genotype TTG, which was associated with higher logical memory scores in the APOE $\varepsilon 4$ positive subset of LBC1936. Again, the individual responsible for the interaction result did not carry the haplotype associated with increased logical memory scores (APP region 2 genotype, TTG; individual genotype, CT-TT-TT).

The two BIN1 SNPs involved in the association of $A P P-B I N 1$ with verbal declarative memory (rs10200967 and rs4663098) are located near to the $5^{\prime}$ end of the BIN1 gene. There is high LD in this region of BIN1, and the SNP associated with LOAD in the recent GWAS, rs744373, is located $21580 \mathrm{bp} 5^{\prime}$ of $\operatorname{rs} 4663098\left(D^{\prime}=0.93\right)$. There are no current reports of an in vivo interaction between BIN1 and $A P P$. However, $A P P$ is a transmembrane protein and is transported through the secretory pathway. It is possible that through its role in endocytosis, BIN1 may interact with APP.

\section{Conclusions}

This study indicates that gene specific variation and genegene interactions may influence cognition. Our strongest results implicate a role for a haplotype at the TRAPPC6A locus in non-verbal reasoning in individuals lacking the $A P O E \& 4$ allele. A less clear role for APP and BIN1 in influencing verbal declarative memory in individuals carrying at least one $A P O E \varepsilon 4$ allele is suggested.

The effect sizes we have observed in this study are small. Indeed, despite the comparability of genomic LD structure, the majority of these associations were not replicated in the LBC1921 cohort. However, it should be noted that the replication cohort $(n=505)$ is smaller than the discovery cohort $(n=998)$. Particularly, our main results were observed in the smaller APOE stratified groups. In addition, the individuals in each cohort were retested at different ages; the LBC1921 were re-tested at age 79, while the LBC1936 were re-tested at age 70, and not all cognitive tests used were all identical, although they were similar.

The results presented here were obtained with SNPs not previously associated with sporadic AD, suggesting that either allelic heterogeneity or a functional SNP is not yet identified (Table 4). Nonetheless, the results presented here identify interactions between recently identified and previously known $\mathrm{AD}$ genes and provide an interesting insight into potential molecular pathways underlying cognitive traits. They require further investigation in larger identically phenotyped cohorts.

\section{Acknowledgments}

G. Hamilton is an Alzheimer's Society Fellow. The authors thank the cohort participants who contributed to these studies. Genotyping of the cohorts was supported by the UK's Biotechnology and Biological Sciences Research Council (BBSRC). Phenotype collection in the Lothian Birth Cohort 1921 was supported by the BBSRC, The Royal Society, and The Chief Scientist Office of the Scottish Government. Phenotype collection in the Lothian Birth Cohort 1936 was supported by Research Into Ageing (continues as part of Age UK's The Disconnected Mind project). The work was supported by The University of Edinburgh Centre for Cognitive Ageing and Cognitive Epidemiology, part of the cross council Lifelong Health and Wellbeing Initiative (G0700704/84698). Funding from the BBSRC, EPSRC, ESRC, and MRC is gratefully acknowledged.

\section{References}

[1] K. A. Welsh, N. Butters, J. P. Hughes, R. C. Mohs, and A. Heyman, "Detection and staging of dementia in Alzheimer's disease: use of the neuropsychological measures developed for the Consortium to Establish a Registry for Alzheimer's Disease," Archives of Neurology, vol. 49, no. 5, pp. 448-452, 1992.

[2] M. A. Lambon Ralph, K. Patterson, N. Graham, K. Dawson, and J. R. Hodges, "Homogeneity and heterogeneity in mild cognitive impairment and Alzheimer's disease: a crosssectional and longitudinal study of 55 cases," Brain, vol. 126, no. 11, pp. 2350-2362, 2003.

[3] R. J. Perry and J. R. Hodges, "Attention and executive deficits in Alzheimer's disease. A critical review," Brain, vol. 122, no. 3, pp. 383-404, 1999.

[4] A. Goate, M. C. Chartier-Harlin, M. Mullan et al., "Segregation of a missense mutation in the amyloid precursor protein gene with familial Alzheimer's disease," Nature, vol. 349, no. 6311, pp. 704-706, 1991.

[5] E. I. Rogaev, R. Sherrington, E. A. Rogaeva et al., "Familial Alzheimer's disease in kindreds with missense mutations in a gene on chromosome 1 related to the Alzheimer's disease type 3 gene," Nature, vol. 376, no. 6543, pp. 775-778, 1995. 
[6] R. Sherrington, E. I. Rogaev, Y. Liang et al., "Cloning of a gene bearing missense mutations in early-onset familial Alzheimer's disease," Nature, vol. 375, no. 6534, pp. 754-760, 1995.

[7] W. J. Strittmatter, A. M. Saunders, D. Schmechel et al., "Apolipoprotein E: high-avidity binding to $\beta$-amyloid and increased frequency of type 4 allele in late-onset familial Alzheimer disease," Proceedings of the National Academy of Sciences of the United States of America, vol. 90, no. 5, pp. 19771981, 1993.

[8] D. Harold, R. Abraham, P. Hollingworth et al., "Genomewide association study identifies variants at CLU and PICALM associated with Alzheimer's disease," Nature Genetics, vol. 41, no. 10, pp. 1088-1093, 2009.

[9] J. C. Lambert, S. Heath, G. Even et al., "Genome-wide association study identifies variants at CLU and CR1 associated with Alzheimer's disease," Nature Genetics, vol. 41, no. 10, pp. 10941099, 2009.

[10] S. Seshadri, A. L. Fitzpatrick, M. A. Ikram et al., "Genomewide analysis of genetic loci associated with Alzheimer disease," Journal of the American Medical Association, vol. 303, no. 18, pp. 1832-1840, 2010.

[11] M. M. Carrasquillo, O. Belbin, T. A. Hunter et al., "Replication of CLU, CR1, and PICALM associations with Alzheimer disease," Archives of Neurology, vol. 67, no. 8, pp. 961-964, 2010.

[12] J. J. Corneveaux, A. J. Myers, A. N. Allen et al., "Association of CR1, CLU and PICALM with Alzheimer's disease in a cohort of clinically characterized and neuropathologically verified individuals," Human Molecular Genetics, vol. 19, no. 16, pp. 3295-3301, 2010.

[13] M. I. Kamboh, R. L. Minster, F. Y. Demirci et al., "Association of CLU and PICALM variants with Alzheimer's disease," Neurobiology of Aging. In press.

[14] I. J. Deary, J. Corley, A. J. Gow et al., "Age-associated cognitive decline," British Medical Bulletin, vol. 92, no. 1, pp. 135-152, 2009.

[15] I. J. Deary, W. Johnson, and L. M. Houlihan, "Genetic foundations of human intelligence," Human Genetics, vol. 126, no. 1, pp. 215-232, 2009.

[16] A. Payton, "The impact of genetic research on our understanding of normal cognitive ageing: 1995 to 2009," Neuropsychology Review, vol. 19, no. 4, pp. 451-477, 2009.

[17] N. M. Wisdom, J. L. Callahan, and K. A. Hawkins, "The effects of apolipoprotein $\mathrm{E}$ on non-impaired cognitive functioning: a meta-analysis," Neurobiology of Aging, 2009.

[18] J. Mengel-From, K. Christensen, M. McGue, and L. Christiansen, "Genetic variations in the CLU and PICALM genes are associated with cognitive function in the oldest old," Neurobiology of Aging, vol. 32, no. 3, pp. 554.e7-554.e11, 2011.

[19] I. J. Deary, A. J. Gow, M. D. Taylor et al., "The Lothian Birth Cohort 1936: a study to examine influences on cognitive ageing from age 11 to age 70 and beyond," BMC Geriatrics, vol. 7, article 28, 2007.

[20] I. J. Deary, M. C. Whiteman, J. M. Starr, L. J. Whalley, and H. C. Fox, "The impact of childhood intelligence on later life: following up the Scottish mental surveys of 1932 and 1947," Journal of Personality and Social Psychology, vol. 86, no. 1, pp. 130-147, 2004.

[21] I. J. Deary, M. C. Whiteman, A. Pattie et al., "Ageing: cognitive change and the APOE $\varepsilon 4$ allele," Nature, vol. 418, no. 6901, p. 932, 2002.

[22] I. J. Deary, M. C. Whiteman, A. Pattie et al., "Apolipoprotein E gene variability and cognitive functions at age 79: a follow-up of the Scottish mental survey of 1932," Psychology and Aging, vol. 19, no. 2, pp. 367-371, 2004.

[23] M. Luciano, A. J. Gow, S. E. Harris et al., "Cognitive ability at age 11 and 70 years, information processing speed, and APOE variation: the Lothian Birth Cohort 1936 study," Psychology and Aging, vol. 24, no. 1, pp. 129-138, 2009.

[24] M. Luciano, A. J. Gow, M. D. Taylor et al., "Apolipoprotein E is not related to memory abilities at 70 years of age," Behavior Genetics, vol. 39, no. 1, pp. 6-14, 2009.

[25] M. Lezak, Neuropsychological Testing, Oxford University Press, Oxford, UK, 2004.

[26] D. Wechsler, WAIS-IIIUK Administration and Scoring Manual, Psychological Corporation, London, UK, 1998.

[27] D. Wechsler, WMS-IIIUK Administration and Scoring Manual, Psychological Corporation, London, UK, 1998.

[28] J. Raven, J. Court, and J. Raven, Manual for Raven's Progressive Matrices and Vocabulary Scales, HK Lewis, London, UK, 1977.

[29] D. Wechsler, Wechsler Memory Scale, The Psychological Corporation, San Antonio, Tex, USA, Revised edition, 1987.

[30] L. M. Houlihan, G. Davies, A. Tenesa et al., "Common variants of large effect in F12, KNG1, and HRG are associated with activated partial thromboplastin time," American Journal of Human Genetics, vol. 86, no. 4, pp. 626-631, 2010.

[31] W. J. Kent, C. W. Sugnet, T. S. Furey et al., "The human genome browser at UCSC," Genome Research, vol. 12, no. 6, pp. 996-1006, 2002.

[32] J. Li and L. Ji, "Adjusting multiple testing in multilocus analyses using the eigenvalues of a correlation matrix," Heredity, vol. 95, no. 3, pp. 221-227, 2005.

[33] M. Steffens, T. Becker, T. Sander et al., "Feasible and successful: genome-wide interaction analysis involving all $1.9 \times 10^{11}$ pairwise interaction tests," Human Heredity, vol. 69, no. 4, pp. 268-284, 2010.

[34] S. Purcell, B. Neale, K. Todd-Brown et al., "PLINK: a tool set for whole-genome association and population-based linkage analyses," American Journal of Human Genetics, vol. 81, no. 3, pp. 559-575, 2007.

[35] J. C. Barrett, B. Fry, J. Maller, and M. J. Daly, "Haploview: analysis and visualization of LD and haplotype maps," Bioinformatics, vol. 21, no. 2, pp. 263-265, 2005. 


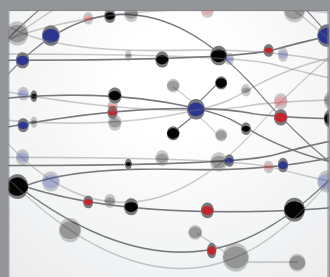

The Scientific World Journal
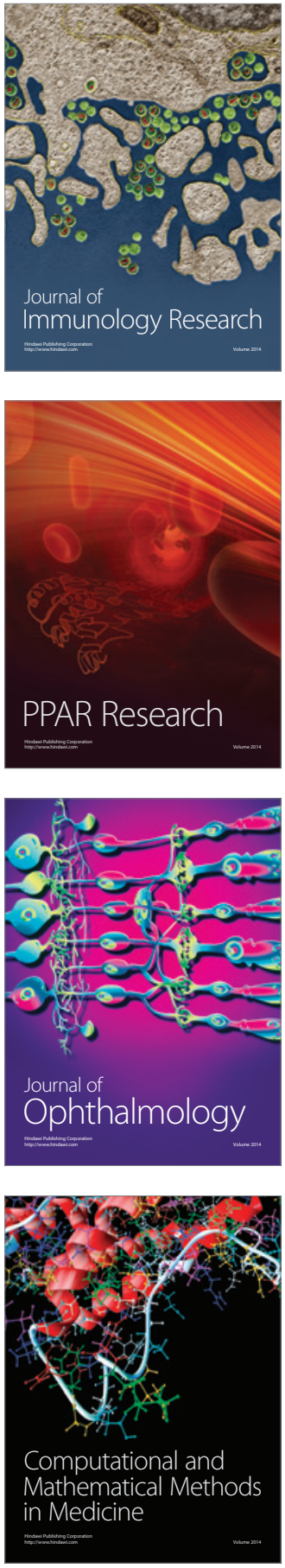

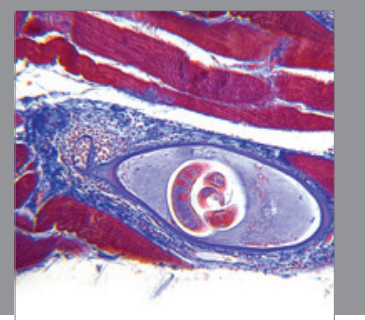

Gastroenterology

Research and Practice
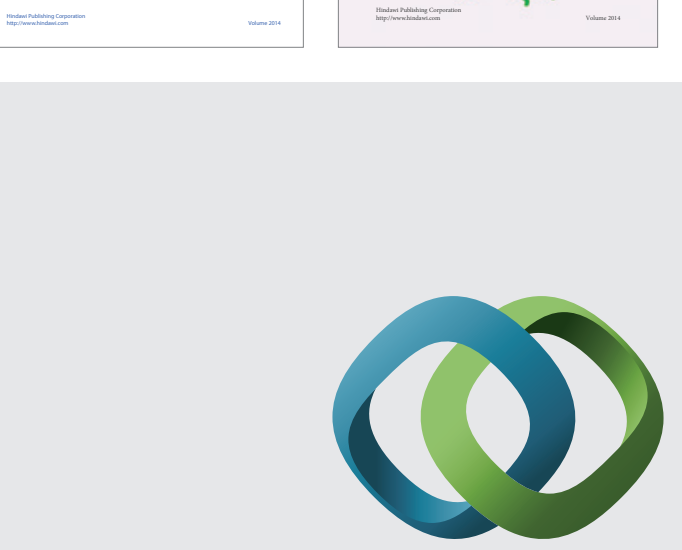

\section{Hindawi}

Submit your manuscripts at

http://www.hindawi.com
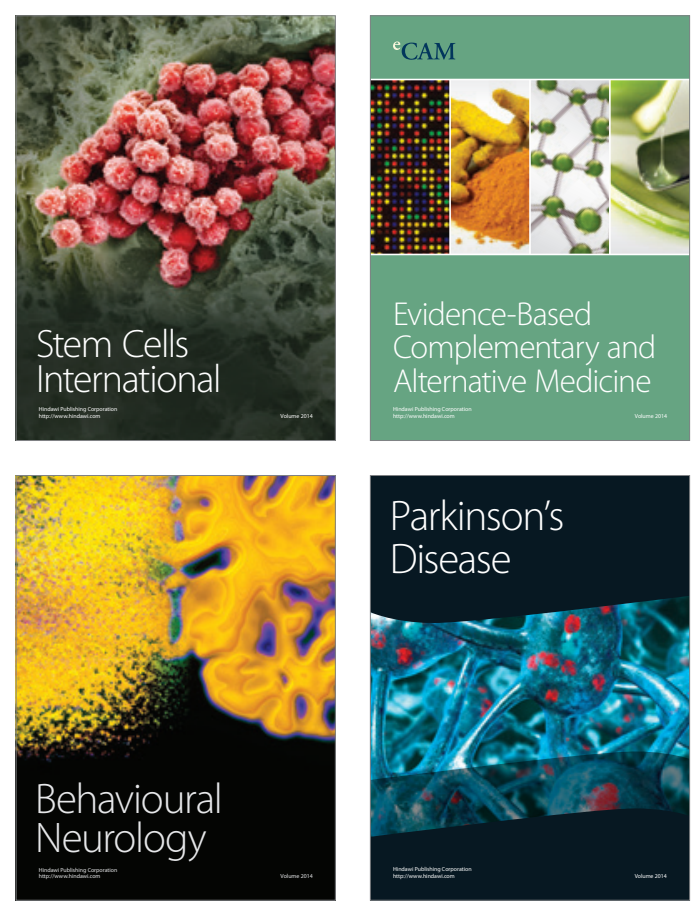

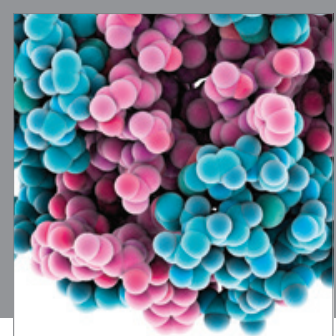

Journal of
Diabetes Research

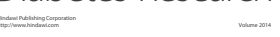

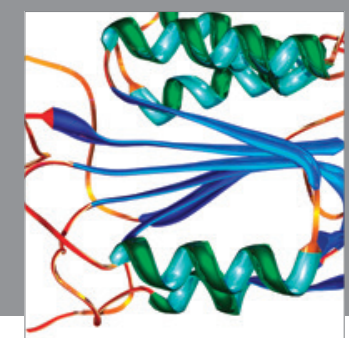

Disease Markers
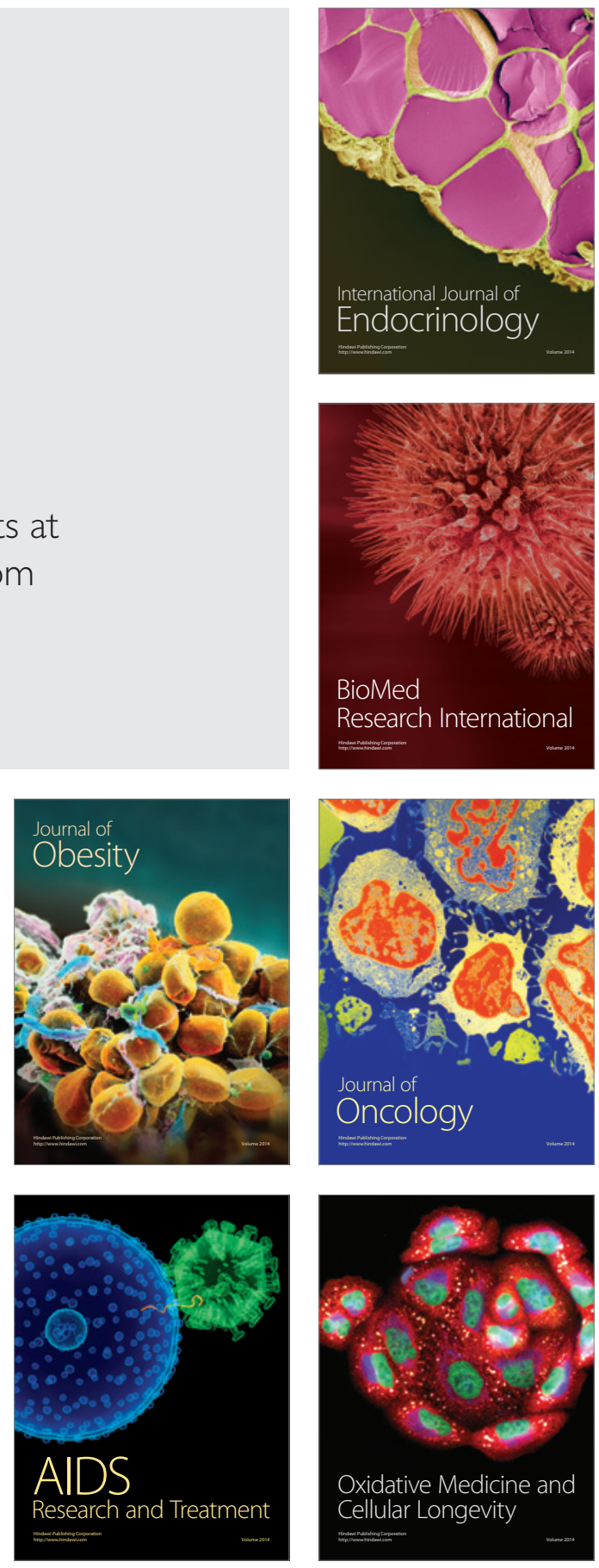